\title{
Morbidity in nocturnal asthma: sleep quality and daytime cognitive performance
}

\author{
M F Fitzpatrick, H Engleman, K F Whyte, I J Deary, C M Shapiro, N J Douglas
}

\begin{abstract}
Most patients with asthma waken with nocturnal asthma from time to time. To assess morbidity in patients with nocturnal asthma nocturnal sleep quality, daytime sleepiness, and daytime cognitive performance were measured prospectively in 12 patients with nocturnal asthma (median age 43 years) and 12 age and intellect matched normal subjects. The median (range) percentage overnight fall in peak expiratory flow rate (PEF) was 22 (15 to 50) in the patients with nocturnal asthma and $4(-4$ to 7$)$ in the normal subjects. The patients with asthma had poorer average scores for subjective sleep quality than the normal subjects (median paired difference $1 \cdot 1$ (95\% confidence limits $0 \cdot 1,2 \cdot 3)$ ). Objective overnight sleep quality was also worse in the asthmatic patients, who spent more time awake at night (median difference 51 (95\% CL 8.1, 74) minutes), had a longer sleep onset latency (12 (10, 30) minutes), and tended to have less stage 4 (deep) sleep $(-33(-58,4) \mathrm{min}$ utes). Daytime cognitive performance was worse in the patients with nocturnal asthma, who took a longer time to complete the trail making tests (median difference $62(22,75)$ seconds) and achieved a lower score on the paced serial addition tests $(-10(-24,-3))$. Mean daytime sleep latency did not differ significantly between the two groups $(2(-3$, 7) minutes). It is concluded that hospital outpatients with stable nocturnal asthma have impaired sleep quality and daytime cognitive performance even when having their usual maintenance asthma treatment.
\end{abstract}

Respiratory Medicine Unit, City Hospital, Edinburgh EH10 5SB M F Fitzpatrick H Engleman K F Whyte N J Douglas

Department of Psychiatry, Royal Edinburgh Hospital, Edinburgh EH10 5HF C M Shapiro

Department of Psychology, University of Edinburgh, Edinburgh EH8 9XE I J Deary

Correspondence to: Dr Douglas

Accepted 22 May 1991 result in impaired daytime cognitive perion may mance. ${ }^{56}$ We postulated that the long term sleep disruption resulting from a combination of nocturnal airway narrowing and drug treatment might cause impairment of daytime cognitive performance in patients with nocturnal asthma. We have therefore measured daytime cognitive performance, nocturnal sleep quality and objective daytime sleepiness in stable patients with nocturnal asthma.

\section{Methods}

We studied 12 patients who were clinically stable but had nocturnal asthma, who had had no hospital admissions or change in medication for at least one month before the study, and 12 normal subjects. Nocturnal asthma was defined as a mean overnight fall in peak expiratory flow rate of at least $15 \%$ in association with at least one awakening a week due to wheezing, coughing, or breathlessness, during a two week run in period, in a patient with known asthma. Each patient was matched with a normal subject for age ( \pm 2 years) and premorbid intelligence on the basis of the national adult reading test ${ }^{7}$ (scores matched to \pm 5 in the national adult reading test). This has been extensively validated as a test of premorbid intellect. ${ }^{89}$ Normal subjects were selected from respondents to a newspaper advertisement requesting healthy volunteers for asthma research. All said that they were non-smokers, were taking no medication, and had no history of respiratory illness. None of the patients or normal subjects had any history of psychiatric illness or sleep related illness or had used sedative medication.

Patients and normal subjects kept a diary card throughout the study period (two weeks) on which they recorded the number of awakenings each night and assigned a score to the subjective sleep quality on a scale from 0 ("very refreshing sleep") to 5 ("didn't sleep at all"). In addition, patients recorded on their diary cards peak expiratory flow measurements (best of three attempts) on four occasions during the day, and during the night if they woke. Normal subjects had their peak expiratory flow (best of three attempts) recorded before and after their sleep studies. Throughout the study patients continued to take all their usual maintenance asthma drugs (inhalers: beta agonist and inhaled corticosteroid-all 12 patients, ipratropium-8, cromoglycate-2; oral medication: theophylline -6 , beta agonist -6 , corticosteroid-5).

Subjects spent at least three consecutive nights at home before the sleep studies and the daytime testing sessions. Each patient underwent full polysomnography ${ }^{10}$ to exclude obstructive sleep apnoea. Each had in addition overnight sleep studies performed on two consecutive nights, when electroencephalography, electro-oculography, and electromyography 
only were performed (with our standard electrode placement ${ }^{11}$ ) so that sleep could be studied with minimum interference. The first of these two sleep studies was used for acclimatisation and only data from the second night were analysed. Sleep scoring was performed without knowledge of the identity of the subject. For objective assessment of daytime sleepiness, multiple sleep latency tests ${ }^{12}$ were performed on all subjects after at least three consecutive nights at home.

On a separate day all subjects underwent a two hour battery of psychometric tests, beginning at $9 \mathrm{am}$. The tests lasted about two hours and consisted of the following: inspection time (a measure of perceptual intake speed) $)^{1314}$; paced auditory serial addition test (PASAT: a test of concentration and attention) ${ }^{15}$; trail making $A$ and $B$ from the Halstead-Reitan battery ${ }^{16}$ (a test of visual scanning, hand-eye coordination, and mental set flexibility); simple reaction time; driving simulation; the Rey auditory verbal learning test (a test of short term memory $)^{17}$; the block design and digit symbol substitution subtests from the Wechsler Adult Intelligence Scale (revised). ${ }^{18}$ Each subject also completed a hospital anxiety and depression scaled questionnaire ${ }^{19}$ on the morning of psychometric testing.

Subjects were asked not to eat or drink anything containing caffeine (coffee, cocoa, cola, chocolate) throughout the study period and to avoid alcohol for at least $\mathbf{4 8}$ hours before the psychometric and sleep tests. Plasma theophylline concentrations were measured four hours after the morning dose on the day of psychometric testing in the patients taking theophylline.

Table 1 Clinical characteristics (median, range) of patients with nocturnal asthma and controls

\begin{tabular}{lll}
\hline & Asthmatic patients & Normal subjects \\
\hline Age & $43,24-68$ & $44,25-69$ \\
Sex & $9 \mathrm{~F}, 3 \mathrm{M}$ & $6 \mathrm{M}, 6 \mathrm{~F}$ \\
NART score $_{\text {PEF (\% pred) }}^{30,21-39}$ & $31,20-37$ \\
Overnight fall in PEF (\%) & $65,46-94$ & $105,97-110$ \\
\hline
\end{tabular}

NART - national adult reading test; PEF—peak expiratory flow.

Table 2 Sleep variables: median differences for mean values for the normal $(N)$ and asthmatic $(A)$ subjects with $95 \%$ confidence limits

\begin{tabular}{lrrrr}
\hline & \multicolumn{2}{l}{ Median difference } & \\
\cline { 2 - 3 } Variable & $N-A$ & $95 \% C L$ & \multirow{2}{*}{$p^{\star}$} \\
\hline Sleep efficiency index (\%) & 7 & 1, & 15 & $<0.05$ \\
Sleep onset latency (min) & -19 & -10, & -30 & $<0.01$ \\
Time spent awake at night (min) & -35 & -8, & -74 & $<0.05$ \\
Time awake after sleep onset (min) & -18 & -57, & 21 & NS \\
Duration (min) of sleep: & -6 & -23, & 5 & NS \\
Stage 1 & -1 & -20, & 26 & NS \\
Stage 2 & 2 & -15, & 10 & NS \\
Stage 3 & 30 & -4, & 58 & NS \\
Stage 4 & 2 & -15, & 43 & NS \\
REM & -5 & -7, & 3 & NS \\
MSLT (mean, min) & -0.9 & $0 \cdot 2,-1.9$ & $<0.05$ \\
Subjective data: & -1.2 & $-0.1,-2.3$ & \\
$\quad$ No of nocturnal wakenings & & & \\
Sleep quality score & &
\end{tabular}

*Wilcoxon signed ranks test. MSLT—multiple sleep latency test.

\section{DATA ANALYSIS}

Wilcoxon signed ranks tests were used to analyse differences between the normal subjects and the asthmatic patients. Results are expressed as the median of the paired differences (values from normal subjects minus those from patients with nocturnal asthma) with $95 \%$ confidence intervals. ${ }^{20}$ Multiple linear regression was used to assess the importance of possible contributory factors to differences between the two groups.

\section{Results \\ METHYLXANTHINE}

Of the six patients claiming to take regular oral theophylline, five had detectable plasma concentrations of theophylline on the day of psychometric testing. Four patients had concentrations within our therapeutic range (8-20 $\mu \mathrm{g} /$ $\mathrm{ml}$ ) and one a subtherapeutic concentration. The median (range) serum theophylline concentration in these five patients was 11 (714) $\mu \mathrm{g} / \mathrm{ml}$.

\section{DIARY CARD DATA}

Patients' characteristics, including peak expiratory flow data, are given in table 1 and data on subjective sleep quality in table 2 . The median score for subjective sleep quality was worse in the patients with nocturnal asthma than in the normal subjects $(p<0.05)$. The median number of self recorded awakenings per night did not, however, differ significantly between the two groups $(p=0 \cdot 13)$.

\section{OBJECTIVE SLEEP QUALITY AND DAYTIME} SLEEPINESS

Sleep efficiency (total time spent asleep $\times 100$ total recording time) was lower in the patients with nocturnal asthma $(p<0.05)$. The asthmatic patients spent longer awake because they took longer to get to sleep than the normal subjects $(p<0.01)$; there was no significant difference in the time spent awake after the onset of sleep between the two groups $(p=0.26)$. The asthmatic group tended to spend less time in stage 4 (deep) sleep than the normal subjects $(p=0.06)$.

Multiple sleep latency test results showed no difference in average daytime sleep latency between the two groups $(\mathrm{p}=0 \cdot 12)$.

\section{DAYTIME COGNITIVE PERFORMANCE}

Significant differences between the asthmatic and normal subjects were noted in the paced auditory serial addition and trail making tests (table 3). The total PASAT score (score for parts A and B combined, maximum 120 ) was higher in the normal subjects than in the asthmatic subjects $(p<0.03)$. This was due to a worse performance by the asthmatic patients on the more demanding half of the paced auditory serial addition test (part B, two second interval between numbers: $p<0.02$ ). The patients with nocturnal asthma also took longer to complete the trail making tests than the normal subjects $(p<0.01)$ because they performed worse on the more demanding half of the test (trail B, in which a series of alternate 
Table 3 Psychometric performance:-median differences for mean values for the normal $(N)$ and asthmatic ( $A$ ) subjects (with $95 \%$ confidence limits)

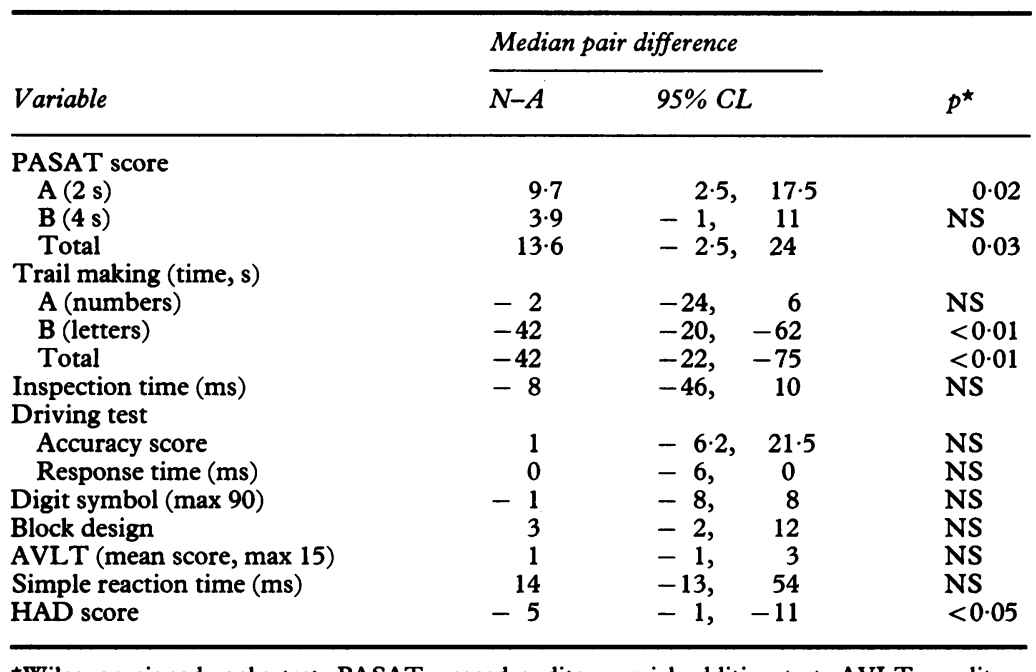

*Wilcoxon signed ranks test. PASAT-paced auditory serial addition test; AVLT -auditory verbal learning test; $\mathrm{HAD}$-hospital anxiety and depression score.

numbers and letters has to be joined together: $(\mathrm{p}<0.01))$. The asthmatic group had higher scores on the hospital anxiety and depression scale than the normal subjects $(p<0.05)$, seven of the asthmatic group having a score of 11 or more, suggesting possible depression, compared with three of the normal subjects; this difference was not, however, significant $\left(\chi^{2}\right.$ test: $\mathrm{p}=0.09$ ).

\section{FACTORS CONTRIBUTING TO THE DIFFERENCE} BETWEEN ASTHMATIC AND NORMAL SUBJECTS When the five patients with detectable plasma theophylline concentrations and the paired control subjects were excluded from the analysis, significant impairments in many of the cognitive performance and sleep variables remained, despite the fact that data on only seven pairs remained for analysis (trail making B: $p<0.03$; trail making $A+B: p<0.03$; PASAT B: $p<0.06$; subjective sleep quality: $\mathrm{p}=0.05$; sleep onset latency: $\mathrm{p}<0.04$.

Data on the 24 subjects were further analysed by multiple linear regression with cognitive performance and sleep time as dependent variables and age, national adult reading test score, hospital anxiety and depression score, use of maintenance theophylline (yes/ no), and nocturnal asthma (yes/no) as independent variables. Nocturnal asthma was the only independent factor influencing performance significantly in part $B$ of the paced auditory serial addition test $(p=0.04)$ and part $B$ of the trail making test $(p<0.01)$. Nocturnal asthma was the strongest factor influencing time spent awake at night $(p=0.15)$ and sleep onset latency $(p=0.08)$, but none of these individual factors had a significant independent influence on time spent awake at night or sleep onset latency. Theophylline intake did not have a significant independent influence on any of the variables found to show impairment in the asthmatic subjects (PASAT $B: p=0.81$; trail making $B: p=0.44$; time spent awake at night: $\mathrm{p}=0.38$; or sleep onset latency: $\mathrm{p}=\mathbf{0 . 7 7}$.

\section{Discussion}

Asthma is a common condition and the prevalence is increasing. ${ }^{21}$ Most asthmatic patients wake with nocturnal symptoms from time to time and patients with more severe asthma may experience asthma symptoms most nights. This study shows that patients who are clinically stable and have nocturnal asthma have poorer daytime cognitive performance and poorer subjective and objective sleep quality than do normal subjects. These abnormalities occurred despite the fact that the number of self reported nocturnal awakenings was no different in patients and normal subjects.

We have shown deficits in patients with nocturnal asthma by comparison with age and intellect matched normal subjects in tests that examine (1) concentration and attention (paced auditory serial addition test) and (2) visual scanning, hand-eye coordination, and mental set flexibility (trail making). These tests are demanding and require information to be stored in working memory while new information is assimilated and responses are made. Detecting small differences in cognitive ability is difficult because most people can call on reserve capacity, even when fatigued. Nevertheless, the paced auditory serial addition and trail making tests, by demanding close attention, mental manipulation and quick thinking, did detect differences between the two groups in the present study. A test of memory (auditory verbal learning test), which was not timed, and tests of driving and reaction time, which are mentally less demanding, did not separate the patients and controls. Our findings extend those of a study that reported that children with "severe asthma" had poorer psychomotor function than non-asthmatic children, ${ }^{24}$ though that study contained no objective measurement of the severity of airflow obstruction, no characterisation of nocturnal asthma, and no sleep recordings.

Possible reasons for the observed deficits in daytime cognitive performance in asthmatic patients include sex differences between patients and controls, sleep deprivation, drug treatment, and depressed mood. Patients and controls were rigorously matched for age and premorbid intelligence, both of which influence psychometric performance. As such matching was difficult to achieve we decided not to match also for sex, as there is no evidence that this affects psychometric performance. Specifically, there is no evidence that gender affects performance in either the paced auditory serial addition $^{15}$ or the trail making tests. ${ }^{23}$

Short term sleep deprivation may lead to demonstrable impairment of daytime cognitive performance. ${ }^{56}$ Furthermore, intermittent disruption of sleep for two nights, amounting to no more than one hour's sleep loss a night, has been found to impair cognitive performance to much the same extent as one night of sleep deprivation. ${ }^{24}$ The cumulative effect of sleep disruption over many nights could therefore account for the deficit in daytime cognitive performance in the patients with nocturnal asthma.

Theophylline has been suspected of causing 
impaired daytime cognitive performance in asthmatic children, ${ }^{25-27}$ but its effect on performance is controversial ${ }^{2829}$ and inadequately documented in adults. Only five of our 12 patients had detectable plasma theophylline concentrations. Most of the important differences between the two groups remained when these five pairs of subjects were excluded from the analysis, and multiple linear regression did not show a significant independent influence of theophylline on psychometric performance. Furthermore, a recent study showed no acute or medium term effect of therapeutic doses of theophylline on the psychometric performance of healthy adults (the test battery included trail making and paced auditory serial addition tests).

Psychomotor retardation is a well recognised feature of clinical depression and, though none of our asthmatic patients had ever been recognised as clinically depressed, their higher scores on the hospital anxiety and depression scale suggest a possible mood effect on their performance. The hospital anxiety and depression score was not, however, a significant independent predictor of either paced auditory serial addition test scores or trail making times according to multiple linear regression analysis: regression of the paired differences in paced auditory serial addition scores and trail making time against paired differences in hospital anxiety and depression scores showed that when the anxiety and depression score difference was zero the estimated mean PASAT score and trail making time differences were significant (PASAT B: $p<0.01$ ); trail making B: $\mathrm{p}<0.03)$.

We have shown for the first time that patients with stable asthma and nocturnal symptoms have impaired sleep quality while continuing to take their usual maintenance antiasthma drugs. Montplaisir and colleagues ${ }^{30}$ showed that eight patients who had attacks of nocturnal asthma while having a sleep recording had less efficient sleep and spent more time awake after the onset of sleep than age matched normal subjects-or they themselves after two weeks of intensive treatment for their asthma. In this study, however, bronchodilators were withheld for six hours before bedtime; patients with asthmatic attacks were asked to perform forced expiratory manoeuvres during the recording night before and after nebulised salbutamol (a process taking "10 to 15 minutes"), assessment of sleep quality was made with subjects wearing full polysomnography equipment, and sleep times were not standardised. Kales et $a l^{31}{ }^{32}$ found that asthmatic children had a shorter total sleep time and spent less time in stage 4 sleep than non-asthmatic children. In these studies, however, (1) bronchodilators were again withheld for at least six hours before bedtime; (2) a group of retrospective control subjects was used; (3) asthma was documented only by symptoms and not by objective measurements of airway obstruction; and (4) sleep times were not standardised. We have found from a single night's recording, without withholding treatment, less efficient sleep, more time spent awake at night, and a trend towards less stage 4 sleep in patients with stable nocturnal asthma than in normal subjects of the same age.

Drug treatment may also affect sleep quality. In nine clinically stable patients with nocturnal asthma theophylline reduced the total sleep time, increased the time spent in drowsiness and light sleep, and reduced the amount of slow wave (deep) sleep ${ }^{4}$ despite reducing nocturnal bronchoconstriction. Administration of an oral beta agonist to patients with asthma did not improve sleep quality despite a reduction in overnight bronchoconstriction. ${ }^{34}$

Depression can cause sleep disturbance. Very depressed patients will typically have a shorter REM latency, a higher proportion of wakefulness and light sleep, and less slow wave sleep with earlier final awakening than normal subjects. ${ }^{34}$ Although seven of our 12 patients had hospital anxiety and depression scores compatible with mild depression, the number was not significantly different from that in our normal subjects, and there was no relation between hospital anxiety and depression scores and daytime performance.

We conclude that stable hospital outpatients with nocturnal asthma, taking their usual maintenance medication, have impaired daytime cognitive performance and impaired subjective and objective sleep quality by comparison with age and intellect matched normal subjects.

This research was supported by a grant from the Asthma Research Campaign.

1 Turner-Warwick M. Epidemiology of nocturnal asthma. Am J Med 1988;85(suppl 1B):6-8.

2 Woolcock AJ, Read J. Lung volumes in exacerbations of asthma. Am J Med 1966;41:259-73.

3 Hetzel MR. Airway function and reactivity at night. In: Barnes PJ, Levy J, eds. Nocturnal asthma. London: Royal Society of Medicine, 1984:59-68. International Congress and Symposium Series, No. 73.

4 Rhind GB, Connaughton JJ, McFie J, Douglas NJ, Flenley DC. Sustained release choline theophyllinate in nocturnal asthma. BMJ 1985;291:1605-7.

5 Williams HL, Lubin A, Goodnow JJ. Impaired performance with acute sleep loss. Psychol Monogr 1959;73:1-26.

6 Friedman RC, Bigger JT, Kornfield DS. The intern and sleep loss. N Engl J Med 1971;285:201-3.

7 Nelson HE. National adult reading test (NART): test manual. Windsor: NFER-Nelson: Windsor, 1982.

8 Nelson HE, O'Connell A. Dementia: the estimation of premorbid intelligence levels using the adult reading test. Cortex 1978;14:234-44.

9 Crawford JR, Stewart LE, Cochrane RHB, Foulds J, Besson JAO. Construct validity of the National Adult Reading Test: a factor analytic study. Personal and Individual Differences 1989;10:793-6.

10 Gould GA, Whyte KF, Rhind GB, Airlie MAA, Catterall JR, Shapiro CM, et al. The sleep hypopnoea syndrome. Am Rev Respir Dis 1988;137:895-8.

11 Catterall JR, Douglas NJ, Calverly PMA, Brash HM, Brezinova V, Shapiro CM, et al. Irregular breathing and hypoxaemia during sleep in chronic stable asthma. Lancet 1982;i:301-4.

12 Carskadon MA, Dement WC, Mitler MM, Roth T, Westbrook PR, Keenan S. Guidelines for the multiple sleep latency test (MSLT): a standard measure of sleepiness. Sleep 1986;9:519-24.

13 Vickers D, Nettelbeck T, Wilson RJ. Perceptual indices of performance: the measurement of "inspection time" and performance: the measurement of "inspection time" and

14 Vickers D, Smith PL. The rationale for the inspection time index. Personal and Individual Differences 1986;7:609-24.

15 Gronwall D. Paced auditory serial addition test: a measure of recovery from concussion. Perceptual and Motor Skills 1977;44:367-73.

16 Boll TJ. The Halstead-Reitan neuropsychological battery. In: Filskov SB, Boll TJ, eds. Handbook of clinical neuropsychology. New York: Wiley, 1981:577-607.

17 Lezak MD. In: Lezak MD, ed. Neuropsychological assessment. New York: Oxford University Press, 1983:422-8. 18 Wechsler D. Manual for the Wechsler adult intelligence scale 
[revised]. New York: Psychological Corporation, 1981.

19 Zigmond AS, Snaith RP. The hospital anxiety and depression scale. Acta Psychiatr Scand 1983:67:361-70.

20 Gardner MJ, Altman DG. Statistics with confidence. London: British Medical Association, 1989:77.

21 Smith JM. The prevalence of asthma and wheezing in children. BrJ Dis Chest 1976;70:73-7.

22 Dunleavy RA, Baade LE. Neuropsychological correlates of severe asthma in children 9-14 years old. $J$ Consult Clin severe asthma in children $1980 ; 48: 214-9$.

23 Reitan RM. Trail making test results for normal and brain damaged children. Perceptual and Motor Skills 1971; 33:575-81.

24 Rachelefsky GS, Wo J, Adelson J, Mickey MR, Spector SL Katz RM, Siegel SC, et al. Behaviour abnormalities and poor school performance due to oral theophylline use. Pediatrics 1986;78:1133-8.

25 Bonnet $M H$. Effect of sleep disruption on sleep, performance and mood. Sleep 1985;8:11-19.25.

26 Springer C, Goldenberg B, Ben DOV I, Godfrey S. Clinical physiologic, and psychologic comparison of treatment by cromolyn or theophylline in childhood asthma. J Allergy Clin Immunol 1985;76:84-9.

27 Furukawa CT, Shapiro GG, Bierman CW, Kraemer MJ, Ward DJ, Pierson WE. A double blind study comparing the effectiveness of cromolyn sodium and sustained release theophylline in childhood asthma. Pediatrics 1984;74: 453-9.

28 Rappaport L, Coffman H, Guare R, Fenton T, De Graw C Twarog F. Effects of theophylline on behaviour and learning in children with asthma. Am J Dis Child 1989; 143:368-72.

29 Creer TL, Gustafson KE. Psychological problems associated with drug therapy in childhood asthma. $J$ Pediatr 1989;115:850-5.

30 Montplaisir J, Walsh J, Malo JL. Nocturnal asthma: Features of attacks, sleep and breathing patterns. Am Rev Respir Dis 1982;125:18-22.

31 Kales A, Beall GN, Bajor GF, Jacobson A, Kales JD. Sleep studies in asthmatic adults: relationship of attacks to sleep stage and time of night. $J$ Allergy 1968;41:164-73.

32 Kales A, Kales JD, Sly RM, Scharf MB, Tan TL, Preston TA. Sleep patterns of asthmatic children: all night EEG studies. J Allergy 1970;46:300-8.

33 Stewart IC, Rhind GB, Power JT, Flenley DC, Douglas NJ. Effects of sustained release terbutaline on symptoms and sleep quality in patients with nocturnal asthma. Thorax 1987;42:797-800.

34 Reynolds CF, Coble PA, Kupfer DJ, Shaw DH. Depressive patients and the sleep laboratory. In: Guilleminault C, ed. Sleeping and waking disorders: indications and techniques. Menlo Park, California: Addison Wesley, 1982:245-64. 